\title{
Statistical study of isolated and non-isolated AGNs in the Local Universe
}

\author{
Nadiia Pulatova ${ }^{1}$, Irina Vavilova ${ }^{2}$, and Peter Berczik ${ }^{2}$ \\ ${ }^{1}$ Crimean Astrophysical Observatory, \\ Nauchnyj, AR Crimea, 98409, Ukraine \\ email: nadya@mao.kiev.ua \\ ${ }^{2}$ Main Astronomical Observatory, NAS of Ukraine, \\ Akademika Zabolotnogo St., 27, Kyiv, MSP-03680, Ukraine \\ email: irivav@mao.kiev.ua, berczik@mao.kiev.ua
}

\begin{abstract}
We present the main results of statistical study of general properties of isolated AGN hosts and AGN hosts in pairs at $z<0.05$ as concerns with the environmental influence on the nuclear galaxy activity. With this aim we compiled the sample of 61 isolated AGNs selected from 2MIG Catalog and Catalog Veron+2010, and the sample of 109 AGNs in pairs selected from NED database.

We found a significant excess content of Sy 2 type galaxies in comparison with Sy 1 type galaxies in both samples (44\% and $11 \%$ among isolated AGNs, $48 \%$ and $6 \%$ among AGNs in pairs, respectively). Isolated AGN hosts of all spectral types are located, in average, at the more far distances and have a more complicated structure than AGNs in pairs as well as they are mostly of late morphological types. Our statistical analysis shows that the observed nuclear activity of isolated 2MIG AGNs during last 3 billions years and more, first of all, is connected with the internal galaxy parameters (relative mass/size, dark/visible matter content, multiwavelength properties of gas-dust medium in accretion disks, tor's structure, central black hole mass etc.).
\end{abstract}

Keywords. Galaxies, active galaxy nuclei, isolated galaxies, Local Universe

\section{Introduction}

The influence of galaxies interaction on activity of its nuclei was studied since 1980-ies and results are not ambiguous till now. The first statistical study of the environment of Seyfert galaxies (both types) was conducted by Petrosian (1982), who found that Sy2 galaxies have an excess of nearby companion galaxies with respect to field galaxies. In recent years a more attention is given to the study of the role of close interactions as a possible triggering mechanism of the AGN activity. Alonso et al. (2008) performed a statistical analysis of AGN hosts in close pairs and in isolation. They argued that isolated AGNs with active black holes could have a merger in recent past as well as a feeding the black hole and star formation rate have been raised during a secular AGN evolution (about $30 \%$ of close galaxy pairs host AGNs, and $23 \%$ are isolated AGNs). Koulouridis et al. (2011), when studying the close and the large scale environment of Sy1, Sy2 and bright IRAS galaxies, concluded that a close interaction is capable even to activate a secular evolution where a normal galaxy becomes first a star-burst, then a Sy2, and finally a Sy1. In our works (Chesnok et al. (2010), Chesnok (2010), Li et al. (2012), Chesnok et al. (2009)) we noticed that nuclear activity is connected, first of all, for isolated AGNs, with the internal galaxy parameters (relative mass, dark/visible matter content, properties of gaseous medium in accretion disks, tor structure, black hole mass) as well as could be affected by the companion's tidality in far past. 
Table 1. Main properties of isolated AGNs (top) and AGNs in pairs (bottom).

\begin{tabular}{|c|c|c|c|c|c|c|c|c|}
\hline Sp.Type & Number & |Morph. | & $\mathrm{Vr}$ & $\mathbf{r}["]$ & $2 \mathrm{~s}$ & $\mid \mathbf{K s} \mathbf{s}[\mathrm{mag}]$ & bar & ring \\
\hline $\begin{array}{l}\text { Sy } 1 \\
\text { Sy } 2 \\
\text { LINERs } \\
\text { AGN } \\
\text { Sy } 1 \mathrm{n} \\
\text { Sy } 1 \mathrm{~h}\end{array}$ & $\begin{array}{c}7(11 \%) \\
27(44 \%) \\
16(26 \%) \\
4(6,5 \%) \\
2(3 \%) \\
2(3 \%)\end{array}$ & $\begin{array}{c}2.9 \\
2.7 \\
2.6 \\
2 \\
3 \\
1\end{array}$ & $\begin{array}{l}6871.3 \\
6328.6 \\
8633.9 \\
7098.5 \\
8388.5 \\
1412.5\end{array}$ & $\begin{array}{c}30.6 \\
28.4 \\
29.64 \\
26.3 \\
19.1 \\
56.9\end{array}$ & $\left|\begin{array}{c}82.05 \\
78.73 \\
80.49 \\
86.5 \\
70.6 \\
131.2\end{array}\right|$ & $\begin{array}{c}10.5 \\
10.38 \\
10.62 \\
10.8 \\
11.51 \\
7.1\end{array}$ & $\left|\begin{array}{c}7(100 \%) \\
15(56 \%) \\
6(38 \%) \\
2(50 \%) \\
2(100 \%) \\
1(50 \%)\end{array}\right|$ & \begin{tabular}{|c}
$5(71 \%)$ \\
$12(44 \%)$ \\
$6(38 \%)$ \\
$2(50 \%)$ \\
$2(100 \%)$ \\
$1(50 \%)$
\end{tabular} \\
\hline $\begin{array}{l}\text { Sy } 1 \\
\text { Sy } 2 \\
\text { LINERs } \\
\text { Sy } 1 \mathrm{~h} \\
\text { Sy } 1.5\end{array}$ & $\begin{array}{c}6(6 \%) \\
53(48 \%) \\
24(22 \%) \\
8(7 \%) \\
9(8 \%)\end{array}$ & $\begin{array}{c}0.7 \\
2.7 \\
1 \\
1.5 \\
0.9\end{array}$ & $\begin{array}{l}4033 \\
4367 \\
3100 \\
1422 \\
6060\end{array}$ & $\begin{array}{l}- \\
- \\
- \\
- \\
-\end{array}$ & $\begin{array}{l}- \\
- \\
- \\
- \\
-\end{array}$ & $\begin{array}{c}9.98 \\
9.14 \\
10.62 \\
9.28 \\
9.93\end{array}$ & $\left|\begin{array}{c}2(3 \%) \\
26(49 \%) \\
8(33 \%) \\
1(49 \%) \\
7(78 \%)\end{array}\right|$ & $\begin{array}{c}1(2 \%) \\
15(28 \%) \\
5(21 \%) \\
1(49 \%) \\
4(45 \%)\end{array}$ \\
\hline
\end{tabular}

\section{Isolated and Non-isolated AGNs: Samples and Properties}

Following our previous research, we undertaken a statistical study of properties of isolated AGNs and AGNs in pairs at $z<0.05$. The sample of 61 isolated AGN hosts was created in the way of a cross-correlation of isolated galaxies from 2MIG Catalog by Karachentseva et al. (2010) with AGNs from Catalog by Veron-Cetty (2010) and of a detailed inspection of each common object. Karachentseva's galaxy isolation criterion applied for 2MIG Catalog, allows us to say that AGNs from our sample were in isolation about 2-3 billions years and more. The sample of 109 AGNs in pairs was selected from NED database. We analyzed activity class, magnitudes in various bands, morphological type including peculiarities (bars and rings), angular diameter, radial velocity and other parameters for isolated AGNs (see, for details, Vavilova \& Pulatova (2012)) and compared its with those of AGNs in pairs to define properties, which are affected by encounter processes. The main results are given in Table 1.

We found a significant excess content of Sy 2 type galaxies in comparison with Sy 1 type galaxies in both samples (44\% and $11 \%$ among isolated AGNs, $48 \%$ and $6 \%$ among AGNs in pairs, respectively). AGNs of Sy2 type in pairs are brighter (Ks-mag

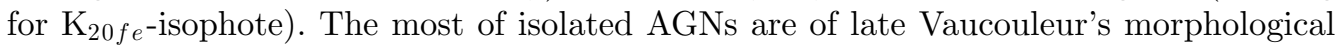
type (ellipticals are absent in general). They are located, in average, at the more far distances, and have a more complicated structure (bars, rings) than AGNs in pairs that could be evidence of tidality effects in their far past. There is no significant difference among isolated AGNs by andle radius (r) and Karachentseva's isolation parameter (2s), excluding 4 galaxies of Sy1n, Sy1h types. So, our statistical analysis is in favour of that the observed processes in the isolated AGN hosts could be fully described by internal properties of galaxies (if by merging then in very far past).

This work is partially supported in frame of the Project F049.2/157 of the SFFR of Ukraine and the Target Scientific-Research Program No.299 of the MAO NAS of Ukraine.

\section{References}

Sol Alonso, M., Lambas, D. G., Tissera, P., \& Coldwell, G. 2008, arXiv:astro-ph/0701192v1

Chesnok, N. G., Sergeev, S. G., \& Vavilova, I. B. 2009, Kinemat. Phys. Celest. Bodies, 25, 107

Chesnok, N., Sergeev S. \& Vavilova, I. 2010, AIP Conference Series, 1206, 328

Chesnok, N. G. 2010, Kosm. Nauka i Teth., 16, 77

Karachentseva, V. E., Mitronova, S. N., Melnyk, O. V. et al. 2010, Bull. SAO, 65, 1

Koulouridis, E., Plionis, M., Chavushyan, V. et al. 2011, arXiv:1111.4084v1

Li, S., Liu, F., Berczik, P. et al. 2012, Astrophys. J., 748, 65

Petrosian, A. R. 1982, Astrofizika, 18, 548

Vavilova, I. B. \& Pulatova, N. G. 2012, MNRAS, in preparation

Veron-Cetty, M. P. 2010, A\&A, 510, V10 